\title{
INTERSTELLAR POLYCYCLIC AROMATIC COMPOUNDS AND ASTROPHYSICS
}

\author{
DOUGLAS M. HUDGINS \\ Astrochemistry Laboratory, MS 245-6, NASA Ames Research Center, \\ Mountain View, CA 94035-1000, USA
}

Over the past fifteen years, thanks to significant, parallel advancements in observational, experimental, and theoretical techniques, tremendous strides have been made in our understanding of the role polycyclic aromatic compounds (PAC) in the interstellar medium (ISM). Twenty years ago, the notion of an abundant population of large, carbon rich molecules in the ISM was considered preposterous. Today, the unmistakable spectroscopic signatures of PAC - shockingly large molecules by previous interstellar chemistry standards - are recognized throughout the Universe. In this paper, we will examine the interstellar PAC model and its importance to astrophysics, including: (1) the evidence which led to inception of the model; (2) the ensuing laboratory and theoretical studies of the fundamental spectroscopic properties of PAC by which the model has been refined and extended; and (3) a few examples of how the model is being exploited to derive insight into the nature of the interstellar PAC population.

$\underline{\text { Keywords }}$ matrix-isolation; infrared spectroscopy; interstellar molecules; polycyclic aromatic molecules and ions

\section{INTRODUCTION}

The cosmic history of the elements carbon, nitrogen, and oxygen - the most abundant, chemically reactive elements after hydrogen - begins with their nucleosynthesis deep within the interiors of late-type stars. These elements are dredged up and thrown off into the surrounding interstellar medium (ISM) during the periods of intense mass-loss that punctuate the end of a typical star's lifecycle. If the abundance of carbon exceeds that of oxygen in these outflows, a wide array of organic materials is formed. The inventory of carbon-rich compounds which arise under such conditions 
includes simple molecules ( $\approx 10$ atoms) such as acetylene, and the polyacetylenic and cyanopolyacetylenic chains $\left[\mathrm{H}(\mathrm{C} \equiv \mathrm{C})_{n+1} \mathrm{H}\right.$ and $\left.\mathrm{H}(\mathrm{C} \equiv \mathrm{C})_{n} \mathrm{C} \equiv \mathrm{N}, n \geq 1\right]$; large, robust polycyclic aromatic molecules composed of tens to hundreds of atoms; and small $(100-1000 \AA)$ amorphous carbon dust particles containing several thousands of atoms or more. As the ejecta disperses, the surrounding ISM is gradually enriched with these carbonaceous materials. In the ISM, these compounds and particles are further modified through a variety of physical and chemical processes including: UV irradiation; cosmic ray bombardment; gas-phase chemistry; and destruction by shock waves generated by supernova explosions. Numerous reviews relevant to this wide range of phenomena can be found in the scientific literature.[1,2]

While refractory dust particles and large molecular aromatic compounds are relatively immune to destruction in the ISM, the simpler polyatomic molecules quickly dissociate under the harsh interstellar UV radiation field, surviving only within the sheltering confines of opaque, "dark" or "dense" interstellar clouds. Within such clouds, the interstellar ultraviolet radiation field is strongly attenuated. Moreover, at the low temperatures which characterize these clouds $(\approx 10-50 \mathrm{~K})$, the majority of molecular species are frozen out upon the surfaces of cold, refractory grains (e.g. [3]). Under such conditions, the interstellar aromatic molecules are further modified by a combination of gas phase and gas-grain surface reactions, and by in-situ, solid-state reactions driven by the attenuated interstellar UV radiation which penetrates the cloud, UV from internal sources within the cloud, and cosmic ray bombardment.[4]

It is within cold, dark molecular clouds such as this that new stars and planetary systems are born. Once part of a molecular cloud becomes unstable under its own gravitational field, it will begin to collapse, forming a protostar. As this collapse proceeds, the angular momentum possessed by the infalling material draws it into a disk. Planetary systems are thought to coalesce from the remnants of this protostellar accretion disk after the young star springs to life (the "Solar Nebula"). Thus, the raw material from which planetary systems form contains aromatic materials in the same diverse states of molecular complexity found in the parent molecular cloud. Indeed, this diverse family of compounds likely represents the largest reservoir of organic material available in these developing planetary systems and, as such, may play a crucial role in the origin and evolution of living systems.

In this paper we review the foundations of the interstellar polycyclic aromatic hydrocarbon (PAH) model and summarize the current state of knowledge regarding the astrophysical implications of these, the largest, most complex organic molecules in the interstellar medium. In section 2, the evidence implicating the presence of polycyclic aromatic compounds (PAC) in the ISM is reviewed. Section 3 provides an overview of the laboratory studies that have been carried out to verify and refine the model. Finally, in section 4, the salient astrophysical implications of this laboratory 
work are presented and insights into the size distribution, structure, and ionization state as a function of interstellar object type are drawn based on the latest astronomical data.

\section{INTERSTELLAR PAC: THE OBSERVATIONAL FOUNDATION}

The discovery of an unexpected infrared emission feature at $890 \mathrm{~cm}^{-1}(11.2$ $\mu \mathrm{m}$ ) from two carbon-rich circumstellar nebulae by Gillett, Forrest, and Merrill [5] marked the beginning of an exciting chapter of modern astrophysics. Gillett et al. recognized that this band was associated with interstellar "dust" and that its identification could give important insight into the formation and evolution of that material through the latter stages of the stellar life cycle. Moreover, this feature could not be reconciled with a simple population of pure graphitic particles such as was at the time believed to arise in such outflows. Subsequent observations by a host of observers (c.f. [6]) revealed that this was just one member of a prominent and ubiquitous family of emission bands whose other components include conspicuous features at 3050,1610,1310, and $1165 \mathrm{~cm}^{-1}(3.3,6.2,7.7$, and $8.6 \mu \mathrm{m}$ ) as well as a complex array of minor bands, plateaus, and underlying continua. Those observers found that the brightest emission came from dusty regions exposed to intense ultraviolet radiation. This spectrum is now known to be an integral part of the IR emission from many different astronomical objects representing all stages of the lifecycle of matter in our galaxy.[7] Moreover, these features carry as much as 20$40 \%$ of the total IR luminosity from many objects [8], indicating that the carrier represents an abundant component of the ISM.

It was first proposed in the mid-1980's that this widespread emission spectrum might be diagnostic of gas phase PAH molecules and closely related species. $[9,10]$ The rationale underlying this suggestion is straightforward. First, the emission bands are non-thermal in nature - that is, they are observed even in regions where the dust temperature is too low for the material to be emitting thermally.[9-11] This, together with the emission-line nature of the spectrum (rather than a smooth continuum) and its correlation with ambient UV flux points to an origin in an ensemble of gas phase molecules, stochastically heated by the absorption of individual UV/visible photons. Second, there is a direct correlation between carbon abundance and the intensity of the emission features [12], implying that the gas phase carriers are carbon-based molecules. Third, the emission features are observed even from extremely harsh environments, indicating that the gaseous, carbon-rich molecules are exceptionally stable. Finally, the positions of the interstellar emission features provide insight into the chemical nature of the material from which they originate. Significantly, prominent bands in the interstellar emission spectrum fall at all the positions that would be expected to arise from the vibrational transitions of 
aromatic molecules: $3050 \mathrm{~cm}^{-1}, \mathrm{CH}$ stretching; $1610 \mathrm{~cm}^{-1}, \mathrm{CC}$ stretching; $1310 \mathrm{~cm}^{-1}$, CC-stretching/CH in-plane bending; $1165 \mathrm{~cm}^{-1}, \mathrm{CH}$ in-plane bending; and $890 \mathrm{~cm}^{-1}, \mathrm{CH}$ out-of-plane bending. Taken together, these elements provide strong evidence that PAHs, are prevalent in the ISM.

Before examining the experimental studies that have been undertaken in this area, it is important in this venue to clarify the working definition of the term "PAH" as it is generally applied within the context of the astrophysical problem. Strictly speaking, the designation "PAH" refers exclusively to compounds containing only carbon and hydrogen. Such a definition is, however, excessively restrictive for an environment as varied and complex as the ISM - an environment whose chemical population may include:

- aromatic compounds which incorporate heteroatoms, particularly nitrogen and oxygen, in their structures.

- aromatic compounds which carry functional groups.

- aromatic compounds which are partially dehydrogenated or superhydrogenated (i.e. $\mathrm{H}_{\mathrm{n}}$-PAHs [13])

- Clusters of aromatic compounds or other 3 dimensional aromaticdominated polymeric structures.

All of these materials must be considered legitimate candidates for one region or another within the ISM until there is solid evidence ruling them out. Although the designation "polycyclic aromatic compounds or PAC" is technically more precise, the term "PAH" has traditionally been expanded to cover all of these species in the astronomical context. Consequently, within the literature on this topic, the terms "PAH" and "PAC" are for all intents and purposes interchangeable.

\section{INTERSTELLAR PAC: THE LABORATORY CHALLENGE}

To more effectively test and exploit the interstellar PAH model and to capitalize on the wealth of astronomical IR spectral data now available requires data on the physical, spectral, and chemical properties of PAC in their likely interstellar forms - free, gas phase neutral molecules and ions, molecular fragments, de-hydrogenated, super-hydrogenated, and heterocyclic variants, etc. $[9,10,14,15]$ Beyond simply reflecting the physical characteristics of the emission zones, such data provide a unique window on the chemical evolution of carbonaceous material throughout the ISM. For example, despite the general similarity between the emission spectra of different objects (i.e. implying a similar class of chemical carrier), the spectra are not identical. On the contrary, significant variations in spectral detail are observed and belie differences in the emitting PAC population from region to region. [16] Thus, models of the interstellar emission which probe the nature of the PAC population and can 
distinguish between the compositions in different regions hold the promise to provide insight into the chemical make-up of different objects; to trace the chemical evolution of those objects as they change from one stage to another; and to probe the ionization balance and other conditions within the emission zones over the wide range of objects which emit the features. Nevertheless, to gain this valuable insight, such models require a thorough understanding of the spectroscopic properties of PAC - fundamental molecular information which can only be obtained through appropriate laboratory experiments and high-level quantum chemical calculations.

Unfortunately, early testing and exploitation of the interstellar PAC model was severely hampered by a general lack of knowledge of the spectroscopic properties of PAC under astrophysically relevant conditions. At the time of its inception, the laboratory data available to the PAC model were limited primarily to spectra measured from pure crystals, salt pellet dispersions, organic solutions, or glassy melts. Under such conditions, the individual PAC molecules are not effectively isolated and interact strongly with each other and/or with the surrounding medium. These conditions strongly influence the measured spectra and are far from the cold, isolated conditions encountered in the ISM. Furthermore, there was virtually no data available on the infrared spectroscopy of PAC cations, species which were expected to dominate the emitting population in the ISM.

In response to this need, over the last decade a major research effort has been underway in the Astrochemistry Laboratory at NASA Ames directed towards the measurement of astrophysically-relevant, laboratory infrared spectroscopic data of a wide range of PAC. Because of the refractory nature of most PAC and their often detrimental physiological activity, spectroscopic studies of gas phase PAC and their associated ions present a number of serious practical difficulties. [17] To reduce or avoid these difficulties, we employ matrix isolation absorption spectroscopy for our studies. In this technique, PAC vapor is generated by warming a solid sample in a small test tube mounted on a high vacuum chamber. The vapor effuses from the tube and is co-condensed with an overabundance of argon onto a cryogenically cooled $(10 \mathrm{~K})$ infrared window suspended within the vacuum chamber. In this highly diluted sample, each molecule is isolated from its neighbors and interacts only very weakly with the inert matrix, resulting in a cold, quasi-gas phase condition. PAC ions are generated by subsequent in-situ UV photolysis of the matrix-isolated neutral species and their absorption features distinguished from those of the neutral by comparison of spectra measured before and after photolysis. More complete discussions of the matrix isolation technique and the various experimental methods that have been employed to generate and study the IR spectral characteristics of both neutral and ionized PAC can be found elsewhere.[18] Among its advantages, this technique is extremely efficient. Essentially all of the vaporized material that exits the reservoir tube is incorporated into the matrix-isolated sample. Since only $\sim 100-200 \mu \mathrm{g}$ of matrix-isolated PAC are required for an experiment, samples of only a few 
milligrams provide enough material for many experiments. Clean-up is also greatly simplified, entailing minimal waste and exposure hazard, since what small amount of PAC residue remains after an experiment is effectively confined to the sample window. Additionally, although the matrix-isolation technique is limited to the measurement of absorption spectra, careful modeling together with the latest experimental studies of jet-cooled, gas phase PAC have shown that a simple thermal model is adequate for calculating of the astrophysical emission spectrum of PAC based on their absorption spectra.[19] Finally, regarding the fidelity of argon matrix-isolated vibrational spectra relative to their corresponding gas phase spectra, the latest theoretical [20] and gas phase experimental [21] studies have shown that for PAC and their ions the vibrational frequencies of matrix isolated species typically fall within 5 to $10 \mathrm{~cm}^{-1}$ ( $\lesssim 1 \%$ ) of their corresponding gas phase values.

Using the matrix-isolation technique, we have generated a spectral database which includes the infrared spectra of over 100 neutral, cationic, and anionic PAC ranging in size from $\mathrm{C}_{10}$ to $\mathrm{C}_{48}$ [22] The species currently included in the database are listed in Table 1. Amongst the species currently represented in the dataset are: (1) the thermodynamically most stable PAHs through coronene, $\mathrm{C}_{24} \mathrm{H}_{12}$, the molecules most likely to be amongst the smallest interstellar PAHs; (2) a representative sampling of species from the fluoranthene family, aromatic hydrocarbons which incorporate a five-membered ring in their carbon skeleton; (3) dicoronylene, $\mathrm{C}_{48} \mathrm{H}_{20}$, the largest $\mathrm{PAH}$ studied to date; and (4) a variety of $\mathrm{N}$-heterocyclic PAC ("aza-PAC"). We have also begun the process of making these data readily available to the scientific community on the internet at <http://web99.arc.nasa.gov/ astrochm/pahdata/index.html>. As discussed in the next section, this data, together with that deriving from similar experimental studies conducted by Vala and coworkers at the University of Florida [23] and extensive theoretical studies [24] demonstrates that mixtures of free molecular PAHs, dominated by PAH ions, can accommodate the global appearance of the interstellar emission spectra and the variations of those spectra.

\section{INTERSTELLAR PAC: INSIGHTS INTO THE INTERSTELLAR EMISSION SPECTRUM}

\subsection{The Effect of Ionization on PAC Spectra.}

One of the early important results to emerge from the laboratory and theoretical studies on neutral and ionized PAC is the remarkably dramatic effect ionization has on their infrared spectra.(cf. [22]) This effect is illustrated in Figure 1. The infrared spectra of neutral PAC are dominated by strong features arising from aromatic $\mathrm{CH}$ stretching vibrations near $3050 \mathrm{~cm}^{-1}(3.3 \mu \mathrm{m})$ and $\mathrm{CH}$ out-of-plane bending vibrations between 900 


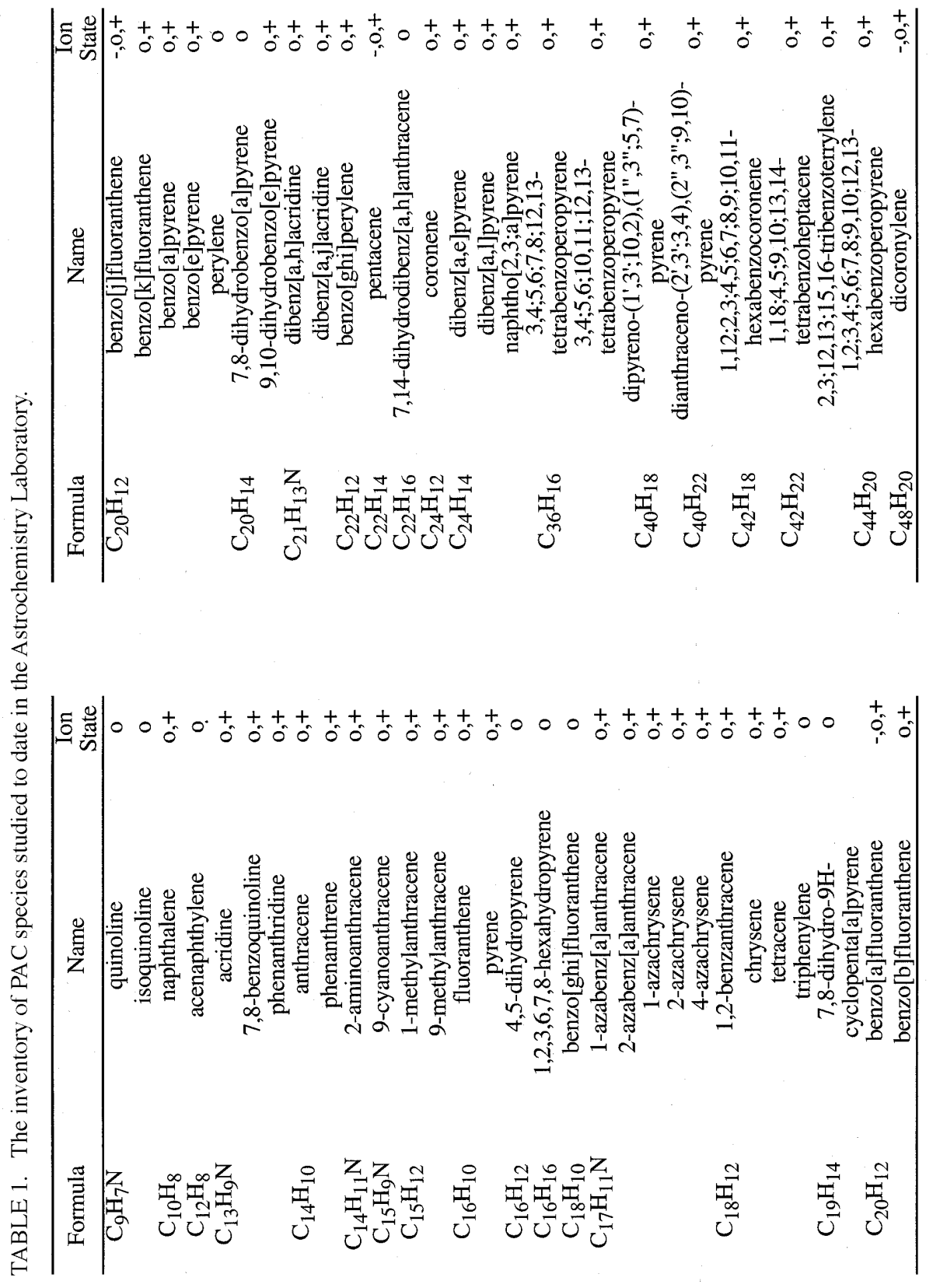




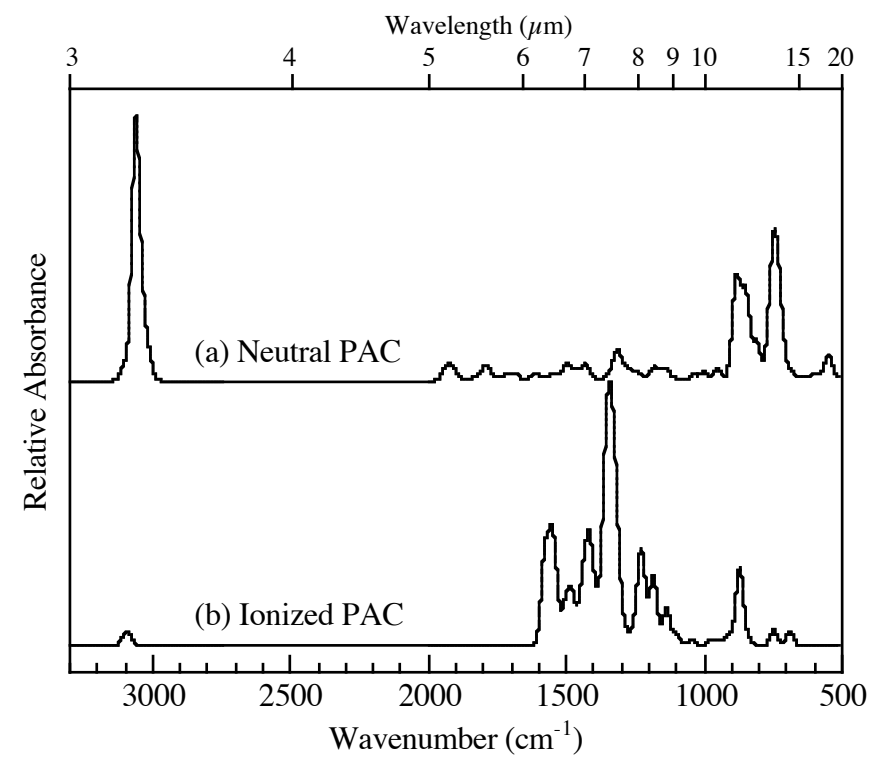

FIGURE 1. A comparison of the absorption spectrum produced by coadding the spectra of the PAC anthracene, tetracene, benz[a]anthracene, chrysene, pyrene, and coronene in (a) their neutral form and (b) their ionized forms. This comparison illustrates that, for PAC, ionization has a much greater influence on relative band intensities than on peak frequencies. The spectra are synthetic representations of the experimental data generated by taking the measured peak positions and relative intensities and assigning each a $30 \mathrm{~cm}^{-1}$ FWHH consistent with molecules emitting under interstellar conditions.

and $700 \mathrm{~cm}^{-1}(11$ and $14 \mu \mathrm{m})$. Weaker features arising from aromatic CC stretching and $\mathrm{CH}$ in-plane bending vibrations are observed in the 1600 to $1100 \mathrm{~cm}^{-1}$ (6 to $9 \mu \mathrm{m}$ ) range. In ionized PAC, on the other hand, the situation is completely reversed. Enhanced by an order of magnitude relative to their neutral counterparts, the 1600 to $1100 \mathrm{~cm}^{-1} \mathrm{CC}$ stretching and $\mathrm{CH}$ in-plane bending modes now dominate the spectra of PAC cations. Conversely, suppressed by an order of magnitude, the $\mathrm{CH}$ stretching features have all but disappeared from the cation spectra. The $\mathrm{CH}$ out-ofplane bending modes are also suppressed in the cations, but much more modestly so $(\approx 2 \mathrm{x})$. As a result, ionization produces a global pattern of band intensities that is in much better agreement with the pattern of intensities observed in the interstellar emission spectrum (see, for example, Figures 2 and 3 below).

\subsection{PAC Models of the Interstellar Emission.}

Within the framework of the PAC model, the interstellar spectrum arises from the combined emission of a complex mixture of PAC. Therefore, to model the appearance of this spectrum one must consider the composite 
spectrum of a variety of different PAC. Thus, with the availability of the extensive database of astrophysically relevant spectra presented above, one can begin to analyze and compare the PAC populations in different emission zones. The following examples serve to illustrate how such an analysis can yield important insight into the nature and properties of the PAC population, and how this information reflects the physical and chemical conditions within the emission regions themselves. A discussion of the simple visual fitting procedure employed can be found in [25]. The laboratory spectra shown in Figure 1 above and Figures 2 and 3 below are synthetic representations of the experimental data generated by taking the measured peak positions and relative intensities and assigning each a 30 $\mathrm{cm}^{-1} \mathrm{FWHH}$ in accordance with that expected of molecules emitting under interstellar conditions (i.e. high levels of vibrational excitation and extremely low pressure).

\subsubsection{PAC in late stellar outflows.}

The protoplanetary nebula phase likely represents the earliest stage in the lifecycle of cosmic PAC.[10,14,26] During the epoch of copious mass loss that punctuates the last stages of a star's life, C, N, and O produced during the final fitful stages of nucleosynthesis deep within the star are dredged up and cast off together with the majority of the dying star's atmosphere. If the abundance of carbon exceeds that of oxygen in this shell, a rich variety of carbon-rich compounds are formed. The object designated IRAS 22272+5435, whose spectrum is shown in Figure 2, is a carbon-rich object undergoing just such a transformation. [27] The observed infrared emission is excited by the remaining, relatively cool central red giant star. Eventually, the outer layers of the star are completely thrown off, exposing the ejecta to the harsh ionizing radiation of the remaining ferociously hot $(\mathrm{T} \sim 50,000$ to $150,000 \mathrm{~K})$ stellar core and ushering in the visually-stunning "planetary nebula" phase.[28] Thus, such an object is enigmatically referred to as the proto-planetary nebula.

A model laboratory spectrum of IRAS $22272+5435$ generated using the ca. 1999 database is also shown in Figure 2.[25] Inspection of the composition of this mixture (given in the figure caption) reveals that it is dominated by neutral PAC $(\sim 60 \%)$ and that it includes species with a broad range of stabilities, from large, condensed PAC (e.g. dicoronylene) to naphthalene, the smallest PAC. Note also that the mixture is internally consistent in that the neutral and cationic forms of the same PAC have been used to construct the fit (i.e. there are no PAC present in ionized form, but not neutral form, and vice-versa). A mixture such as this is certainly reasonable when one takes into consideration the nature of the object. Here, in the region where aromatic compounds are beginning to appear and before they have been exposed to the ferocious radiation field of the coming planetary nebula phase, it is logical to expect that the emitting material would contain a diverse mixture of species, representing a wide range of thermodynamic stabilities. Furthermore, given the relatively 


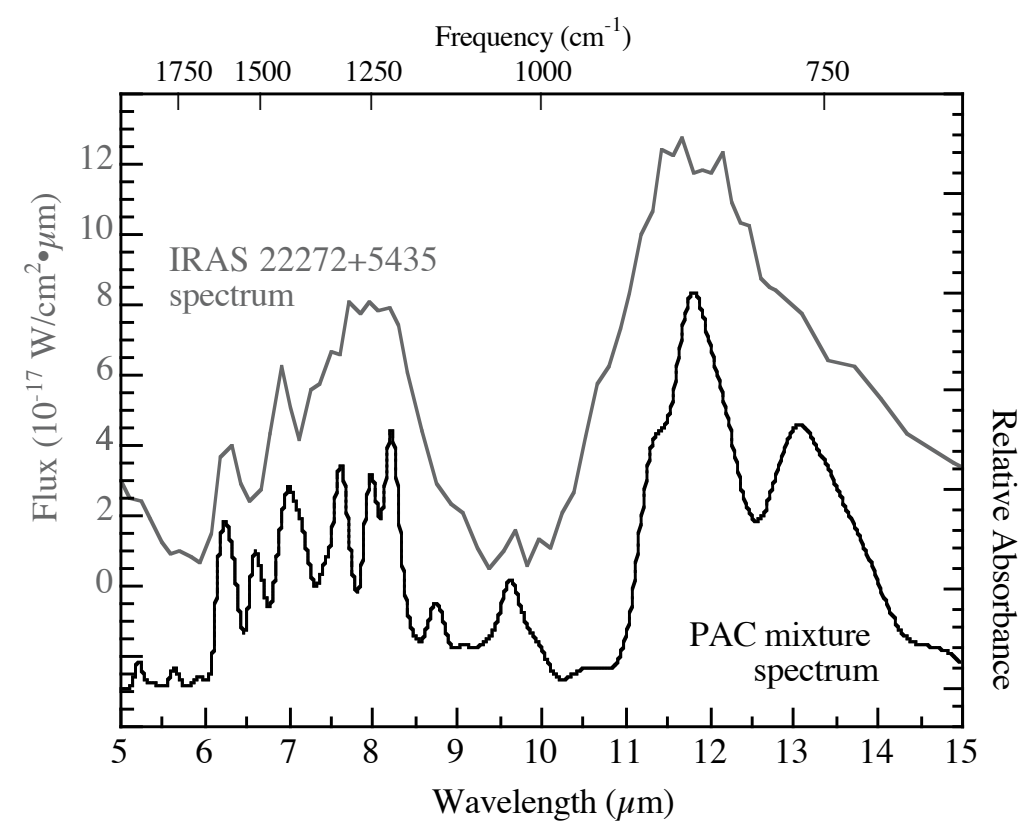

FIGURE 2. Comparison of the infrared emission spectrum of the protoplanetary nebula IRAS $22272+5435$ with a composite absorption spectrum generated by coadding the individual spectra of 19 neutral and cationic PAHs. The mixture is comprised of ("O" indicates a neutral species; "+" indicates a cation; chemical formulae are given in Table 1): $18 \%$ dicoronylene $^{\mathrm{O}}, 14 \%$ each naphthalene $^{+}$and 9,10-dihydrobenzo(e)pyrene ${ }^{+}, \quad 11 \% \quad 9,10$ dihydrobenzo[e]pyrene ${ }^{\mathrm{o}}, 10 \%$ each benzo[j]fluoranthene ${ }^{\mathrm{O}}$ and coronene $^{\mathrm{o}}$, and $3 \%$ each benzo[a]fluoranthene ${ }^{+}$, benzo[j]fluoranthene ${ }^{+}$, coronene ${ }^{+}$, hexabenzocoronene $^{+}$, dicoronylene ${ }^{+}$, benzo[a]fluoranthene ${ }^{0}$, naphthalene ${ }^{\mathrm{o}}$, and hexabenzocoronene ${ }^{\mathrm{o}}$. The spectrum of IRAS $22272+5435$ is adapted from $[27(\mathrm{a})]$.

benign radiation field produced by a $5,300 \mathrm{~K}$ star, it is also expected that both neutral and ionized species should contribute to the emission. The PAC population which provides the fit shown in Figure 2 reflects exactly these characteristics.

\subsubsection{PAC in star-forming regions.}

Next, consider the very different environment represented by the ionization ridge in the Orion Nebula. This region represents the interface where the energetic stellar winds from a cluster of massive, hot young stars impacts the surrounding, interstellar cloud material.[29] The infrared emission spectrum of that region measured recently by the European Infrared Space Observatory (ISO) [30], together with that of the best-fit mixture of species drawn from the current database, is shown in Figure 3. Here the material 


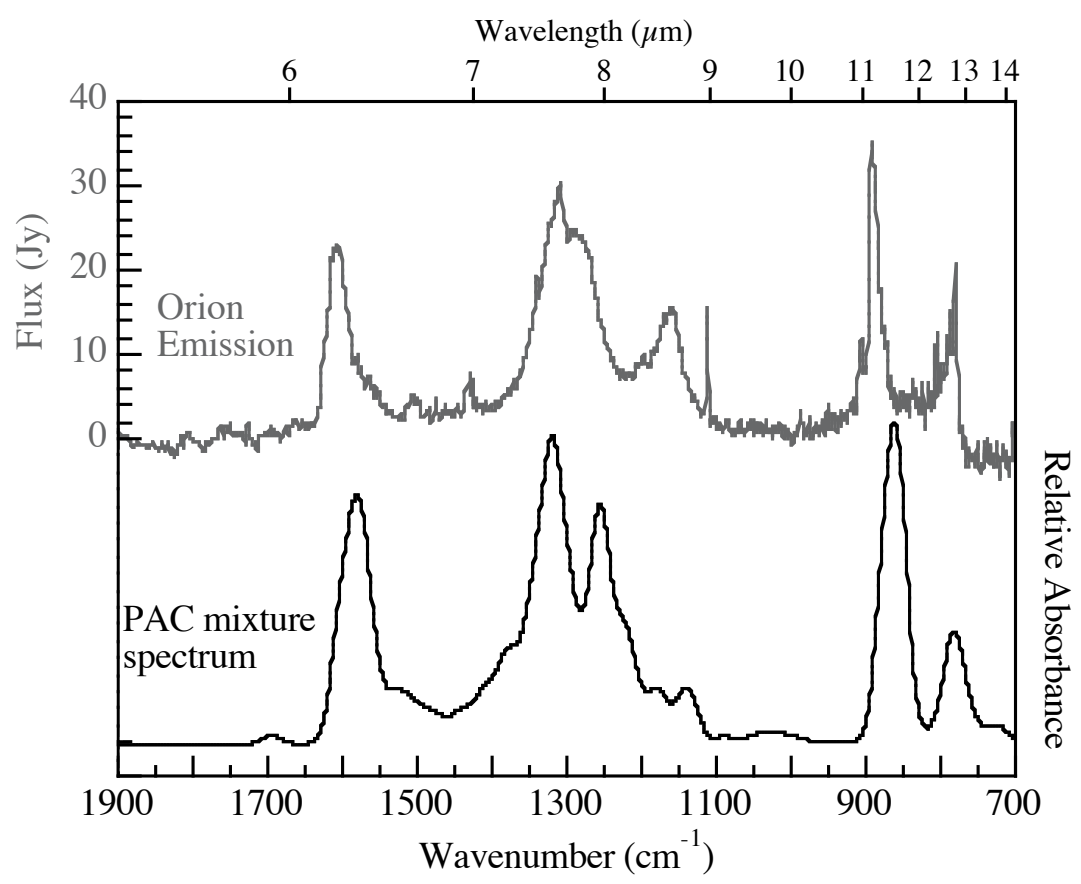

FIGURE 3. Comparison of the infrared emission spectrum of the Orion ionization ridge with a composite absorption spectrum generated by coadding the individual spectra of $11 \mathrm{PAH}$ species. The mixture is comprised of ("O" indicates a neutral species; "+" indicates a cation; chemical formulae are given in Table 1): $22 \% \quad$ coronen $\mathrm{e}^{\mathrm{o}}$; $19 \% \quad 3,4 ; 5,6 ; 10,11 ; 12,13-$ tetrabenzoperopyrene ${ }^{+} ; 15 \%$ coronene $^{+} ; 7 \%$ each of dicoronylene ${ }^{+}$, benzo[b]fluoranthene ${ }^{+}$, benzo[k]fluoranthene ${ }^{+}$, and naphthalene ${ }^{\mathrm{o}} ; 4 \%$ each of naphthalene ${ }^{+}$, phenanthrene ${ }^{+}$, chrysene $^{+}$, and tetracene ${ }^{+}$. The Orion spectrum is adapted from [30].

originally produced in late stellar outflows has been "aged" for perhaps a billion years, alternately bathed in the harsh galactic interstellar radiation field in the so-called "diffuse" interstellar medium and then frozen into interstellar ice particles in opaque "dense molecular clouds". Now this material is being exposed to the ionizing radiation from the adjacent hot young stars forming nearby. The composition of the mixture that provides the best fit to the Orion spectrum (given in the figure caption) is quite revealing about the nature of the PAC population there. Unlike the protoplanetary nebula, where neutral PAC dominated the model mixture, here the composite spectrum contains $70 \%$ ionized species. Furthermore, the role of less stable PAC structures (i.e. less condensed) in the mix is substantially reduced compared to the protoplanetary nebula case. Instead, PAC having more highly condensed (and therefore more thermodynamically favored) structures dominate the emission. In fact, three of the thermodynamically most favored PAHs in the mixture 
contribute more than $60 \%$ of the match to Orion shown in Figure 3. The $\mathrm{PAH}$ population reflected here is again entirely consistent with what one would expect given the nature and history of this object. The molecules found in this region are those which have survived the interstellar gauntlet and the fierce radiation from the nearby stellar association. Lesser stable components of the carbon-rich material initially ejected into the ISM have long since been 'weeded out' - either destroyed or isomerized into more stable structures by energetic processing. In addition, in the presence of the intense ultraviolet radiation from the nearby stars, it is expected that a substantial portion of the molecular population is likely to be ionized. Thus, it is entirely reasonable that we find the best-fit PAC mixture for the Orion ionization ridge reflects a disproportionately large contribution from the hardiest species and from ionized species.

While the PAC mixtures used to provide the spectral fits in the above examples are not unique, within the constraints of the database there is not a lot of variation possible in the choice of the dominant PAC species in each. Since IRAS $22272+5435$ and the Orion ionization ridge represent very different epochs in the evolution of cosmic carbon, the spectral differences reveal how carbonaceous material evolves as it passes from its circumstellar birth site into the general ISM. While there can be great variability in the appearance of the interstellar emission spectrum between objects or from one region to another within one object [18], these differences can readily and naturally be accommodated by different PAC populations. The differences in the astronomical spectra are a direct consequence of differences in the composition of the emitting PAC population. The PAC population, in turn reflects a variety of physical and chemical conditions such as radiation field flux and energy, ionization states, carbon abundance, etc., in the emitting regions. Thus, given the ubiquity and intensity of the interstellar infrared emission features, PAC hold the potential to provide a powerful probe of interstellar environments which span all the stages in the lifecycle of cosmic carbon.

4.3. Drawing Insights from the Models: Constraints on the Interstellar PAC Population.

Closer inspection of Figure 3 also shows that, although the model spectrum reproduces all the major peaks and relative intensities of the Orion spectrum reasonably well, it is not perfect. Nevertheless, differences in detail such as these can also yield insight into the nature of the emitting interstellar PAC population.

As discussed above, the spectra of PAC cations are dominated by features in the $1600-1100 \mathrm{~cm}^{-1}$ spectral region. While in principle a particular PAC cation may exhibit features anywhere throughout this region, it is generally observed that two or three features dominate this region (c.f. [22(b),21(g)]). Furthermore, these dominant features tend to cluster in the vicinity of the strong interstellar 1610 and $1320 \mathrm{~cm}^{-1}(6.2$ and $7.7 \mu \mathrm{m})$ emission features. As a result, when the spectra of a number of 
PAC cations are coadded, two dominant features tend to emerge with an appearance very similar to those of the interstellar spectrum (e.g. Figure 3). Nevertheless, as pointed out above, while the overall agreement between the two spectra in Figure 3 is excellent, careful inspection reveals that the positions of the nominal " $6.2 \mu \mathrm{m}$ " and " $7.7 \mu \mathrm{m}$ " features in the model spectrum are somewhat "pinched" compared to the astronomical spectrum. Close inspection now made possible thanks to the availability of moderate resolution interstellar spectra shows that this arises from the fact that the position of the $1575 \mathrm{~cm}^{-1}$ feature in the composite laboratory spectrum does not precisely match the peak of the interstellar emission band $\left(1610 \mathrm{~cm}^{-1} ; 6.2 \mu \mathrm{m}\right)$. To understand the origin of this discrepancy, it is useful to take a step backward and consider the spectra of the individual cations from which the mixture is derived.

Table 2 contains a list of PAC cations together with the positions of their dominant $\mathrm{CC}$ stretching features. Inspection of these data shows that the measured positions of the dominant $\mathrm{CC}$ stretching feature in the spectra of PAC cations tends to fall as much as 40 to $80 \mathrm{~cm}^{-1}$ lower in frequency than the $1610 \mathrm{~cm}^{-1}$ astronomical feature. As a result, as illustrated in Figure 3, when the spectra in the current database are combined, the dominant feature which emerges in this region also necessarily reflects this frequency difference. Further inspection of the data in Table 2 reveals that the positional discrepancies of the dominant $\mathrm{CC}$ stretching features are not random but are, in fact, size dependent, with the bands of the largest molecules falling closest to the interstellar position. This trend is illustrated

TABLE 2. Positions of the Dominant Aromatic CC Stretching Features for the PAC in the Infrared Spectral Database.

\begin{tabular}{ccc}
\hline PAC Cation & $\begin{array}{c}\text { Number of C } \\
\text { atoms }\end{array}$ & $\begin{array}{c}\text { Dominant CC stretch } \\
\text { Position }\left(\mathrm{cm}^{-1}\right)\end{array}$ \\
\hline naphthalene & 10 & 1526 \\
anthracene & 14 & 1540 \\
phenanthrene & 14 & 1565 \\
pyrene & 16 & 1553 \\
benz[a]anthracene & 18 & 1540 \\
chrysene & 18 & 1560 \\
tetracene & 18 & 1543 \\
benzo[a]fluoranthene & 20 & 1540 \\
benzo[b]fluoranthene & 20 & 1572 \\
benzo[e]pyrene & 20 & 1557 \\
benzo[j]fluoranthene & 20 & 1576 \\
benzo[k]fluoranthene & 20 & 1583 \\
benzo[ghi]perylene & 22 & 1578 \\
coronene & 24 & 1579 \\
hexabenzocoronene & 42 & 1571 \\
dicoronylene & 48 & 1607 \\
\hline
\end{tabular}


in Figure 4 which shows a stick diagram of this region of the spectrum for three representative $\mathrm{PAH}$ cations (pyrene, $\mathrm{C}_{16} \mathrm{H}_{10}{ }^{+}$; ovalene, $\mathrm{C}_{32} \mathrm{H}_{14}{ }^{+}$; and dicoronylene, $\mathrm{C}_{48} \mathrm{H}_{20}{ }^{+}$). In this representation the location of each stick reflects the position of an absorption band, while the length of the stick reflects the relative intensity of that band. The approximate FWHH of the canonical interstellar features are indicated by the shaded regions in the diagram. This figure clearly shows that as molecular size increases, the spacing between the most prominent bands increases. Indeed, for the largest molecule, both prominent bands fall squarely within the envelopes of the interstellar features. This confirms the behavior noted by Langhoff $[21(\mathrm{~g})]$ from theoretical work on a more limited range of PAH sizes $\left(\mathrm{C}_{10} \mathrm{H}_{8}\right.$ up to $\left.\mathrm{C}_{32} \mathrm{H}_{14}\right)$. Given this trend, it is reasonable to suggest that the interstellar $6.2-7.7 \mu \mathrm{m}$ spacing is an indicator of the molecular size of the dominant emitting species. Indeed, using the data set presented in Table 2, it is possible to quantify this relationship.

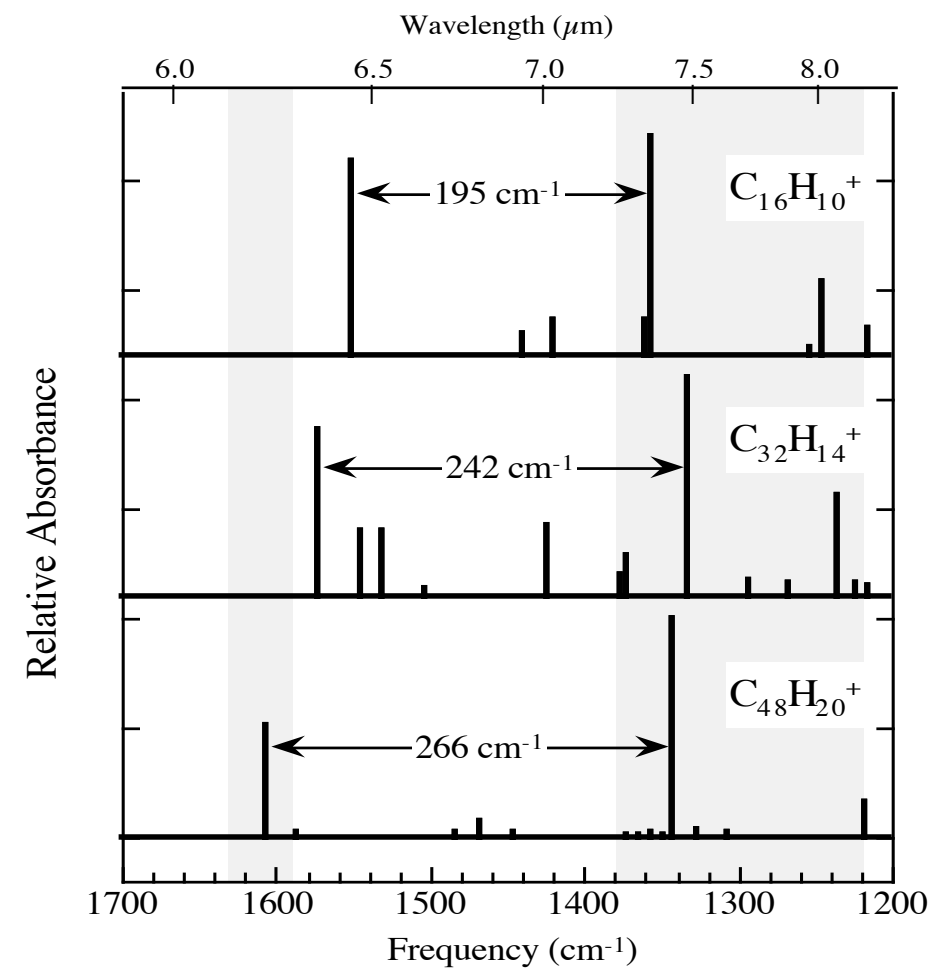

FIGURE 4. A schematic comparison of the absorption spectra of the cations of the PAHs pyrene $\left(\mathrm{C}_{16} \mathrm{H}_{10}{ }^{+}\right)$, ovalene $\left(\mathrm{C}_{32} \mathrm{H}_{14}{ }^{+}\right)$, and dicoronylene $\left(\mathrm{C}_{48} \mathrm{H}_{20}{ }^{+}\right)$ illustrating the evolution of the spacing of the dominant features in the 1700 $-1200 \mathrm{~cm}^{-1}$ region as a function of molecular size. Shaded areas indicate the FWHH of the dominant interstellar emission bands. Data for the ovalene cation taken from $[21(\mathrm{~g})]$. 
Figure 5 shows a plot of the positions of the dominant $\mathrm{CC}$ stretching features as a function of molecular size. Again, the approximate FWHH of the interstellar feature is indicated by shaded bar. A power law fit to these data confirms, as deduced previously from Table 2, that the frequencies of the nominal "6.2 $\mu \mathrm{m}$ " PAC bands increase steadily with size, approaching that of the interstellar band near the largest end of the molecules in the spectral database. Indeed, extrapolation of this trend one finds that the curve intersects the interstellar position at a molecular size of $\approx 60 \mathrm{C}$ atoms. In general, the extrapolation indicates that the dominant $\mathrm{CC}$ stretching features of PAC species should fall within the envelope of the interstellar emission feature for species larger than $\approx 50$ carbon atoms. Moreover, since the average vibrational excitation (and subsequent infrared emission) imparted by the absorption of a given photon decreases with molecular size, it is unlikely that molecules in excess of $80-100 \mathrm{C}$ atoms make a substantial contribution to the emission at these wavelengths. Thus, we conclude that the interstellar $6.2 \mu \mathrm{m}$ feature is dominated by the emission of $50-100 \mathrm{C}$ atom ionized PAC species. While this is consistent with the range of the earlier analyses, these results significantly tighten the constraints on the size of the PAC population that dominates the emission.

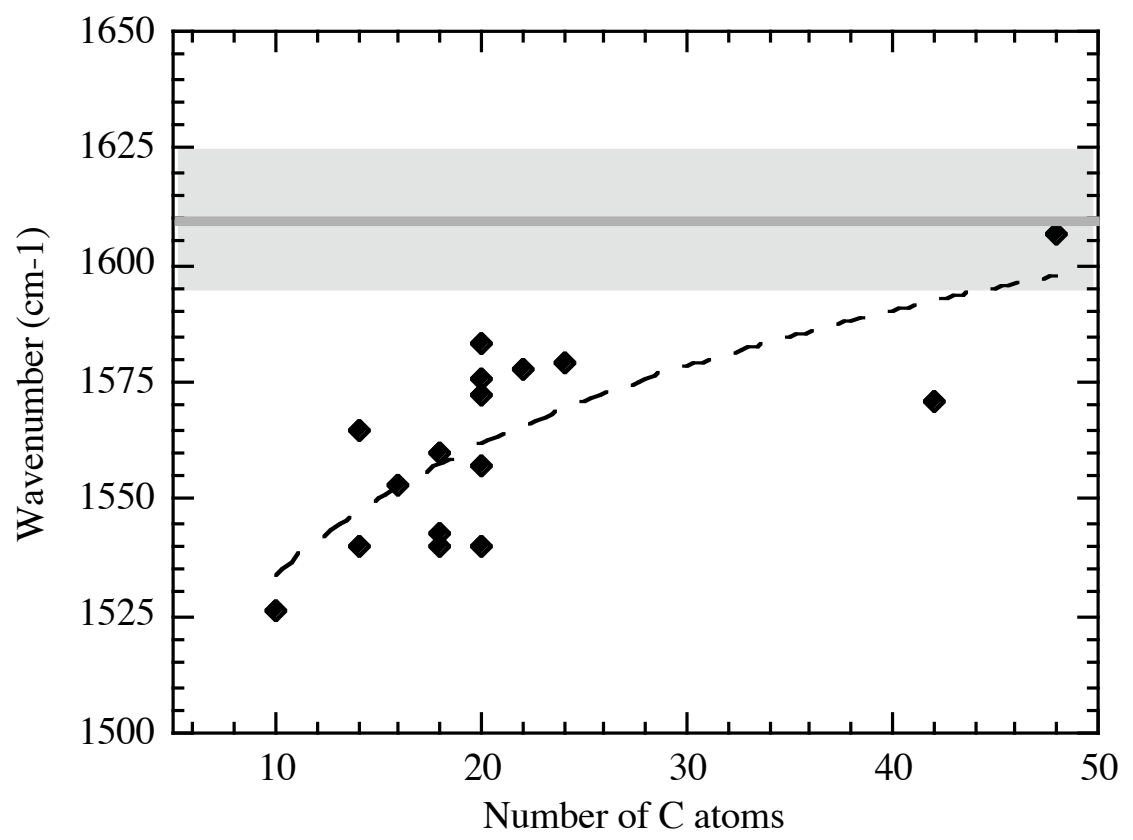

FIGURE 5. A plot of the position of the strongest $\mathrm{CC}$ stretching feature as a function of $\mathrm{C}$ number for the species in Table 2 illustrating the direct relationship between frequency and PAC size. The dotted line represents a power law fit to the data. 
This and other spectroscopic results are beginning to reveal the size, structure, and ionization balance of the interstellar PAC population information which, in turn, reveals much about the disparate conditions in the interstellar emission zones.

\section{CONCLUSION}

The last decade of laboratory infrared spectroscopic studies of PAC has taught us that these species are an integral part of the rich and complex world of Interstellar Chemistry. As recently as the 1960's, it was thought that interstellar conditions were too harsh for any significant polyatomic chemistry to take place. It was thought that any compounds that could form under the extremely low densities of space would quickly be dissociated by the plentiful high-energy radiation. Today we are beginning to see that PAC and related materials are abundant throughout the ISM, and are taking the first steps toward exploiting these species as probes of the physical and chemical conditions in space. The latest high-quality astronomical observations, supported by an ever-increasing database of astrophysically relevant laboratory spectra of PAC and PAC ions, is nowproviding insight into the conditions in IR-emitting regions at an unprecedented level of detail. The I/S PAH model has now moved beyond merely seeking to verify the presence of PAC in space to investigation of the impact of these complex organic species on topics as diverse as interstellar radiative transport to the origin of life. Clearly, PAC hold the potential to be one of the most important and useful classes of interstellar molecules in the coming decades..

\section{References}

[1] (a) Leger, A., d'Hendecourt, L., and Boccara, N. (eds), (1987) PAH and Astrophysics, Proceedings of NATO ASI No.191, D. Reidel Pub. Co., Dordrecht; (b) Allamandola, L.J. and Tielens, A.G.G.M. (eds.), (1989) Interstellar Dust, Proceedings of IAU Symposium 135, Kluwer Academic Pub., Dordrecht.

[2] Pirronello, V., and Krelowski, J. (eds.), (2001) Solid State Astrochemistry, Kluwer Academic Pub., Dordrecht.

[3] Sandford, S.A., and Allamandola, L.J. (1993) Ap.J., 417, 815 and references therein.

[4] Bernstein, M.P., Sandford, S.A., Allamandola, L.J., Gillette, J.S., Clemett, S.J., and Zare, R.N. (1999), Science, 283, 1135.

[5] Gillett, F.C., Forrest, W.J., and Merrill, K.M. (1973) Ap.J., 183, 87. 
[6] (a) Russell, R.W., Soifer, B.T., and Willner, S.P. (1977) Ap J., 217, L149; (b) Aitken, D.K. (1981), in Wynn-Williams C.G., and Cruikshank, D.P. (eds.), Infrared Astronomy, D. Reidel Pub. Co., Dordrecht, p. 207; (c) Willner, S.P. (1984), in Kessler, M.F., and Phillips, J.P., (eds.), Galactic and Extragalactic Infrared Spectroscopy, D. Reidel Publishing Co., Dordrecht, p. 37; (d) Phillips, M.M., Aitken, D.K., and Roche, P.F. (1984) MNRAS, 207, 25.

[7] Special Edition: First Results from ISO (1996) $A \& A, \mathbf{3 1 5}(2)$.

[8] Puget, J.L. (1989) in Allamandola, L.J., and Tielens, A.G.G.M. (eds.), Interstellar Dust, Kluwer Academic Pub., Dordrecht, p. 119.

[9] Leger, A., \& Puget, J.L. (1984) A\&A, 137, L5.

[10] Allamandola, L.J., Tielens, A.G.G.M., and Barker, J.R. (1985) Ap.J., 290, L25.

[11] Sellgren, K. (1984) Ap.J., 235, 138.

[12] (a) M. Cohen et al. (1986) Ap.J., 302, 737; (b) M. Cohen et al. (1989) Ap.J., 341, 246.

[13] Bernstein, M.P., Sandford, S.A., and Allamandola, L.J. (1996) Ap.J., 472, L127;

[14] Allamandola, L.J., Tielens, A.G.G.M., and Barker, J.R. (1989) Ap.J.Supp.Ser., 71, 733.

[15] Puget, J.L., and Leger, A. (1989) Ann.Rev.Astron.Astrophys., 27, 161.

[16] (a) Sloan, G., Grasdalen, G., and LeVan, P. (1993) Ap.J., 409, 412;

(b) Bregman, J., Rank, D., Temi, P., Hudgins, D., and Kay, L. (1993) Ap.J., 411, 794; (c) Bregman, J., Rank, D., Sandford, S., and Temi, P. (1993) Ap.J., 410, 668; (d) Verstraete, L., Puget, J.L., Falgarone, E., Drapatz, S., Wright, C.M., and Timmermann, R. (1996) A\&A, 315, L337; (e) Sloan, G.C., Bregman, J.D., Geballe, T.R., Allamandola, L.J. and Woodward, C.E. (1996) Ap.J., 474, 735; (e) Sloan, G.C., Hayward, T.L., Allamandola, L.J., Bregman, J.D., DeVito, B., and Hudgins, D.M. (1999) Ap.J., 513, L65.

[17] L.J. Allamandola and D.M. Hudgins, in Pirronello, V., and Krelowski, J. (eds.), (2001) Solid State Astrochemistry, Kluwer Academic Pub., Dordrecht.

[18] (a) Hudgins, D., Sandford, S.A., and Allamandola, L.J. (1994) J.Phys.Chem., 98, 4243; (b) Hudgins, D.M., and Allamandola, L.J. (1995) J.Phys.Chem., 99, 3033; (c) Halasinski, T., Hudgins, D.M., Salama, F., Allamandola, L.J., and Bally, T. (2000) J.Phys.Chem., 104, 7484.

[19] (a) Leger, A., d'Hendecourt, L.B., Defourneau, D. (1989), A\&A 216, 148; (b) Schutte, W., Tielens, A.G.G.M., and Allamandola, L.J. (1993) Ap.J., 415, 397; (c) Cook, D.J., and Saykally, R.J. (1998) Ap.J., 493, 793. 
[20] (a) Hudgins, D.M., Bauschlicher, C.W., Jr., Allamandola, L.J., and Fetzer, J.C. (2000) J.Phys.Chem.A, 104, 3655; (b) Bauschlicher, C.W., Jr., Langhoff, S.R., Sandford, S.A., and Hudgins, D.M. (1997) J.Phys.Chem.A, 101, 2414; (c) Langhoff, S.R. Bauschlicher, C.W., Jr., Hudgins, D.M., Sandford, S.A., and Allamandola, L.J. (1998) J.Phys.Chem.A, 102, 1632; (d) Hudgins, D.M., Bauschlicher, C.W., and Allamandola, L.J. (2001) Spectrochim.Acta.A, 57, 907.

[21] (a) Piest, J.A., von Helden, G., and Meijer, G. (1999) Ap.J. 520, L75; (b) Piest, J.A., Oomens, J., Bakker, J., von Helden, G., and Meijer, G. (2001) Spectrochim.Acta A, 57A, 717.

[22] Hudgins, D., Sandford, S.A., and Allamandola, L.J. (1994) J.Phys.Chem., 98, 4243; (b) Hudgins, D.M., and Allamandola, L.J. (1995) J.Phys.Chem., 99, 3033; (c) Hudgins, D.M., and Allamandola, L.J. (1995) J.Phys.Chem., 99, 8978; (d) Hudgins, D.M., and Allamandola, L.J. (1997) J.Phys.Chem., 101, 3472; (e) Hudgins, D.M., and Sandford, S.A. (1998) J.Phys.Chem.A, 102, 329; (f) Hudgins, D.M., and Sandford, S.A. (1998) J.Phys.Chem.A, 102, 344; (g) Hudgins, D.M., and Sandford, S.A. (1998) J.Phys.Chem.A, 102, 353; (h) Mattioda, A., Hudgins, D.M., Bauschlicher, C.W., Jr., and Allamandola, L.J. (manuscript in preparation).

[23] (a) Szczepanski, J., Roser, D., Personette, W., Eyring, M., Pellow, R., and Vala, M. (1992) J.Phys.Chem., 96, 7876; (b) Szczepanski, J., and Vala, M. (1993) Nature 363, 699; (c) Szczepanski, J., and Vala, M. (1993) Ap.J., 414, 179; (d) Szczepanski, J., Chapo, C., and Vala, M. (1993) Chem Phys, 205, 434; (e) Szczepanski, J., Vala, M., Talbi, D., Parisel, O., Ellinger, Y.J. (1993) J.Chem.Phys., 98, 4494; (f) Vala, M., Szczepanski, J., Pauzat, F., Parisel, O., Talbi, D, and Ellinger, Y.J. (1994) J.PhysChem., 98, 9187; (g) Szczepanski, J., Wehlburg, C., and Vala, M. (1995) Chem.Phys.Lett., 232, 221; (h) Szczepanski, J., Drawdy, J., Wehlburg, C., Vala, M. (1995) Chem.Phys.Lett., 245, 539.

[24] (a) Talbi, D., Pauzat, F., Ellinger, Y. (1993) A\&A, 268, 805; (b) de Frees, D.J., Miller, M.D., Talbi, D., Pauzat, F., and Ellinger, Y. (1993) Ap.J., 408, 530; (c) Pauzat, F., Talbi, D., and Ellinger, Y. (1995) A\&A, 293, 263; (d) Pauzat, F., Talbi, D., and Ellinger, Y. (1997) $A \& A, 319$, 318; (e) Pauzat, F., Talbi, D., and Ellinger, Y. (1999) MNRAS, 304, 241; (f) Pauzat, F., and Ellinger, Y. (2001) MNRAS, 324, 355; (g) Langhoff, S.R. (1996) J.Phys.Chem., 100, 2819; (h) Bauschlicher, C.W., and Langhoff, S.R. (1997) Spectrochim.Acta A, 53, 1225; (i) Bauschlicher, C.W. (1998) Chem.Phys., 233, 29; (j) Bauschlicher, C.W., and Langhoff, S.R. (1998) Chem.Phys., 234, 79; (k) Bauschlicher, C.W., (1998) Chem.Phys., 234, 87; (1) Bauschlicher, C.W. (1998) Chem.Phys., 234, 87; (m) Bauschlicher, C.W., and and Bakes, E.L.O. (2000) Chem.Phys., 262, 285. 
[25] Allamandola, L.J., Hudgins, D.M., and Sandford, S.A. (1999) Ap.J., 511, L115.

[26] (a) Gauger, A., Gail, H.P., and Sedlmayr, E. (1990) A\&A, 235, 345; (b) Cherchneff, I., Barker, J.R., and Tielens, A.G.G.M. (1992) Ap.J., 401, 269; (c) Cadwell, B.J., Wang, H., Feigelson, E.D., and Frenklach, M. (1994) Ap.J., 429, 285.

[27] (a) Buss, Jr., R.H., et al. (1993) Ap.J., 415, 250; (b) Kwok, S., Hrivnak, B.J., and Geballe, T.R. (1995) Ap.J., 454, 394.

[28] (a) Balick, B. (1996) Am.Scientist, 84(4); (b) Kwok, S.A. (1996) Sky and Tel., 92, 38; (c) Kwok, S. (1998) Sky and Telescope, 96, 30.

[29] Bregman, J.D., Allamandola, L.J., Tielens, A.G.G.M., Geballe, T.R., and Witteborn, F.C. (1989) Ap.J., 344, 791.

[30] Peeters, E., Hony, S., Van Kerckhoven, C., Tielens, A.G.G.M., Allamandola, L.J., Hudgins, D.M., and Bauschlicher, C.W. (2002) $A \& A$ (submitted). 